\title{
Weighted Guided Image Filtering - A Survey
}

\author{
Nidhi Sen \\ Mtech scholar, \\ Department of \\ Electronics \& Communication Engineering \\ $\mathrm{NRI}$ Institute of information \\ science \& technology \\ Bhopal, India
}

\author{
Akhilesh Jain \\ Asst. Prof., \\ Department of Electronics \& Communication \\ Engineering \\ $\mathrm{NRI}$ Institute of information \\ science \& technology \\ Bhopal, India
}

\author{
Swapnil Jain \\ H.O.D, Department of Electronics \& \\ Communication Engineering \\ NRI Institute of information \\ science \& technology \\ Bhopal, India
}

\begin{abstract}
It is well-known that local filtering-based edge preserving smoothing method suffers from halo artifacts. In this paper, a weighted guided image filter is introduced by incorporating an edge-aware weighting into an accessible guided image filter to address the problem. The WGIF inherit benefits of both global and local smoothing filters in the sense that: 1) the difficulty of the WGIF is $\mathrm{O}(\mathrm{N})$ for an image with N pixels, which is same as the GIF and 2) the WGIF can avoid halo artifact like the existing global smoothing filters. The WGIF is applied for single image detail enhancement, single image mist removal, and fusion of differently exposed images. Investigational results show that the resultant algorithms create images with better visual quality and at the same time halo artifacts can be avoided from appearing in the final images with negligible rise on running times.
\end{abstract}

\section{Keywords}

Edge-preserving smoothing, weighted guided image filter, edge-aware weighting, detail enhancement, haze removal, exposure fusion .

\section{INTRODUCTION}

MOST application in computer vision and computer graphics engages image filtering to restrain and/or remove content in images. plain linear translation-invariant filters with explicit kernels, like mean, Gaussian, Laplacian, and Sobel filters, have been broadly used in image restoration, sharpening/blurring, feature extraction, edge detection etc. Alternatively, LTI filters be capable of implicitly performed by solving a Poisson Equation the same as in high dynamic range (HDR) compression, image matting, image stitching, and gradient domain manipulation. The filtering kernels are absolutely described by means of the inverse of a homogenous Laplacian matrix. The LTI filtering kernels are spatially independent and invariant of image content. But usually one may desire to consider added information from a known guidance image. The prepare work of an isotropic distribution uses the incline of the filtering image itself to direct a diffusion process, steer clear of smoothing edges. The weighted least squares filter utilizes the filtering input (as an alternative of intermediate results, as in) as the guidance, and optimizes a quadratic function, which is the same to anisotropic diffusion with a nontrivial stable state. The guidance image is able to be another image besides the filtering input in several purposes. For illustration, in colorisation the chrominance channels be supposed to not bleed across luminance edges; in image matting the alpha not shiny should capture the thin structures in a combined image; in haze removal the depth layer should be consistent with the outlook. In these cases, we look upon the chrominance/alpha/depth layers as the image to be filtered, and the composite /luminance/scene as the guidance image, correspondingly. The filtering process is attained by optimizing a quadratic rate function weighted by the guidance image. The result is known by solving a large sparse matrix which only depends on the guide. This inhomogeneous matrix implicitly describes a translation-variant filtering kernel While these optimization based approach frequently defer state-of-the-art class, it comes with the price of costly computational time. One more way to take benefit of the guidance image is to openly build it into filter kernels. The two-sided filter, independently wished-for in, and and later indiscriminate in, is perchance the most accepted one of like explicit filters. Its output at a pixel is a weighted average of the in close proximity pixels, where the weights depend on the color /intensity similarities in the guidance image. The guidance image itself can be the filter input or another image. The two-sided filter can smooth small fluctuations and while preserving edges. However this filter is valuable in various states, it may have unnecessary gradient reversal artifacts near edges. The speedy execution of the bilateral filter is also a challenging problem. Latest techniques rely on quantization process to accelerate but may sacrifice accuracy. In this paper, we propose a novel precise image filter called guided filter. The filtering output is nearby a linear transform of the guidance image. On one side, the guided filter has fine edgepreserving smoothing property like the bilateral filter, but it does not experience from the gradient reversal artifacts. On the other side, the guided filter is capable of to be used beyond smoothing: With the help of the guidance image, it can create the filtering output more structured and less smoothed as compared to the input. We notice that the guided filter performs extremely well in a great range of applications, including image smoothing and enhancement, HDR firmness, flash-no-flash imaging, matting, feathering, dehazing, and 
joint up sampling. Moreover, the guided filter naturally has a time (in the number of pixels $\mathrm{N}$ ) non -estimated algorithm for both high resulation images and gray-scale, regardless of the kernel size and intensity range. Classically, our CPU execution achieves $40 \mathrm{~ms}$ per mega-pixel executing grayscale filtering: To the best of our knowledge, this is one of the best edge preserving filters. A beginning description of this paper was published in ECCV ' 10 . It is significance mentioning that the guided filter has witnessed a sequence of new applications since then. The guided filter allows a highquality real-time stereo matching algorithm. An alike stereo technique is proposed independently in the guided filter has also been applied in optical flow assessment, interactive image segmentation, saliency recognition, and illumination rendering. We accept as true that the guided filter has enormous potential in computer vision and graphics, given its ease, effectiveness, and high-quality. We have provided a public code to make easy future studies.

\section{LITERATURE SURVEY}

Zhengguo Li et. al. [1] "Weighted Guided Image Filtering" In this paper, It is identified that local filtering-based edge preserving smoothing method suffer from halo artifacts. In this paper, a weighted guided image filter is initiated by incorporating an edge-aware weighting into an accessible guided image filter (GIF) to deal with the problem. The WGIF inherits benefit of both global and local smoothing filters in the sense that: 1) the difficulty of the WGIF is $\mathrm{O}(\mathrm{N})$ for an image with $\mathrm{N}$ pixels, which is similar as the GIF and 2) the WGIF can keep away from halo artifacts like the present global smoothing filters. The WGIF is applied for single image detail improvement, single image haze deletion, and blend of differently exposed images. A weighted guided image filter is planned in this paper by incorporating an edgeaware weighting into the guided image filter .The WGIF preserves keen boundaries as well as presented global filters, and the difficulty of the WGIF is $\mathrm{O}(\mathrm{N})$ for an image with $\mathrm{N}$ pixels which is almost same as GIF. Because of the simplicity of the WGIF, it has several applications in the field of computational photography and image processing. Mainly, it is useful to study single image aspect enhancement, fusion of differently exposed images, and single image haze removal. Investigational results illustrate that the resulting algorithms can produce images with excellent visual quality as those of global filters, and same time the running times of the planned algorithms are comparable to GIF based algorithms.

Buyue Zhang et. al. [2] "Adaptive Bilateral Filter for Sharpness Enhancement and Noise Removal" In this paper, we tend to present the adaptive bilateral filter $(A B F)$ for sharpness improvement and noise removal. The $\mathrm{ABF}$ sharpen an representation by raise the incline of the edges while not manufacturing overshoot or undershoot. It's an approach to sharpness improvement that's basically different from the unsharp mask (USM). This new approach to slope restitution also differs significantly from previous slope restoration algorithms in this the ABF doesn't involve detection of edges or their direction, or pulling out of edge shape. In the ABF, the edge slope is enhanced by reworking the histogram through a variety of filter with adaptive offset and width. we tend to demonstrate that $\mathrm{ABF}$ works well for both natural pictures and text images. In this paper, we tend to there an adaptive bilateral filter $(\mathrm{ABF})$. The $\mathrm{ABF}$ retains the general kind of the bilateral filter, but contains 2 necessary modifications. First, an offset is introduced to the range filter within the ABF. Second, both and also the width of the range filter in the ABF are locally adaptive. They depend upon the local image structure, classified in line with the output of the Laplacian of Gaussian (LoG) operator applied to the degraded image. The optimal and are estimated in advance throughout the development of the algorithmic rule via an off-line training procedure employing a set of pairs of original and degraded images. During the training procedure, the MSE between the original and reconstructed images is reduced for each class of pixels.

Anush Krishna Moorthy et. al. [3] "A Two-Step Framework for Constructing Blind Image Quality Indices" Now a day's No reference Image Quality Assessment (NR IQA) algorithms usually assume that the distortion affecting the picture is recognized. This is a preventive supposition for realistic applications; since in a majority of cases the distortions the picture are unidentified.We recommend a innovative 2-step framework for no-reference image quality assessment based on natural scene statistics (NSS). Previously trained, the frameworks don't require any knowledge of the distorting process and the framework is modular in that it can be extended to any number of distortions. We describe the framework for blind image quality consideration and a description of this framework - the blind image quality index (BIQI) is evaluated on the LIVE image quality assessment database.

\section{Jiahao Pang et. al. [4] "Improved Single Image Dehazing}

Using Guided Filter" Single image dehazing is challenging because it is massively ill-posed. Haze removal based on dark channel prior is effective, but refining the transmission map with closed-form matting is computationally expensive. Recent work discovered that using guided filter to refine the transmission map is feasible. In this paper, we intricate single image dehazing by combining dark channel prior and guided image filtering in detail. By analyzing the tradeoffs of this approach, we tend to propose an effective scheme to adapt the parameters Experiments and comparisons show that our method generates satisfactory dehazed results with low computation. In this paper, we elaborate single image dehazing by combining dark channel prior and guided image filtering, and then we study several aspects of this approach. Through experiments and analyses, we propose an effective scheme to adapt the patch size of dark channel and the filtering radius of guided filter. The main benefit of using guided filter to refine the transmission lies in its low computational cost; it also generates comparable dehazed results with He et al.'s work. Since guided image filtering is an approximation of soft matting in, this method may fail when the input image contains abrupt depth changes. Fortunately, it turns out that our work performs quite well on many hazy images, its $\mathrm{O}(\mathrm{N})$ time complexity also makes it appealing for many applications.

Pierre Charbonnier et. al. [5] "Deterministic Edge-Preserving Regularization in Computed Imaging" Many image process problems are sick posed and must be regularised. Usually, a roughness penalty is imposed on the explanation. The complexity is to evade the smooth of edges, which are important attributes of the image. In this paper, we tend to $1 \mathrm{st}$ provide conditions for the design of such an edge-preserving regularization. Under these conditions, we tend to show that it's possible to introduce an auxiliary variable whose role is twofold. First, it marks the discontinuities and makes sure their perpetuation from flat. 2nd it makes the criterion halfquadratic. The optimisation is then easier. We tend to propose a deterministic strategy, based on alternate minimizations on the image and also the auxiliary variable. This results in the definition of an ingenious reconstruction algorithmic rule, 
referred to as ARTUR. A number of theoretical property of ARTUR are discuss. Experimental results illustrate the behavior of the algorithmic rule. These results are shown in the field of tomography, but this technique can be applied in a large number of applications in image processing. In this document, we've measured the trouble of edge-preserving Regularization in computed imaging. Our first aim was to make available a united answer to the question, what belongings have to a possible work (or its derivative) satisfied to make sure the maintenance of edges. We've planned a heuristical study of the first-order necessary conditions which LED us to propose 3 conditions for edge preservation.

\section{METHOD}

We are analysis edge-preserving filtering method in this section. We categorize them as implicit/explicit weightedaverage filters and non average ones:

\section{A. Explicit Weighted-Average Filters}

The two-sided filter is perhaps the simplest and most instinctive one among precise weighted-average filters. It computes the filtering output at each pixel as the average of adjacent pixels, weighted by the Gaussian of both intensity and spatial distance. The bilateral filter smooths the image whereas preserving edges. It has been broadly used in noise reduction, HDR compression, multi scale detail decay, and image abstraction. It is generalized to the joint bilateral filter in, where the weights are computed from one more guidance image moderately than the filtering input. The joint bilateral filter is particularly preferential when the image to be filtered is not dependable to provide edge information, e.g., when it is very loud or is an in-between result, such as in flash/no-flash de noising, image up sampling, image deconvolution, stereo matching, etc. The bilateral filter has limits despite its attractiveness. It has been become aware of in that the bilateral filter might experience from "gradient reversal" manufactured article. The cause is that when a pixel has few similar pixels around it, the Gaussian is the efficiency .Abrute-force implementation is $\mathrm{O}(\mathrm{Nr} 2)$ time with kernel radius r. Durand and Dorsey recommend a piece-wise linear depiction and help FFT-based filtering. Paris and Durand formulate the gray-scale bilateral filter as a 3Dimensional filter in a whole variety domain, and down sample this area to speed up if the Nyquist condition is something like true. In the case of package spatial kernels, Weiss proposes an $\mathrm{O}(\mathrm{N}$ logr) time method based on distributive histograms, and Porikli proposes the first $\mathrm{O}(\mathrm{N})$ time method using integral histograms. We point out that constructing the histograms is effectively perform a $2 \mathrm{D}$ spatial filter in the space-range domain with a 1D range filter followed. Under this viewpoint, both and sample the signal along the range domain but do not reconstruct it. Yang proposes another $\mathrm{O}(\mathrm{N})$ time method which interpolates all along the range domain to allocate more aggressive sub sampling. All of the above methods are linearly difficult w.r.t. the numeral of the sample intensities (e.g., number of linear pieces or histogram bins). They require common sampling to attain acceptable speed, but at the expense of quality degradation if the Nyquist condition is harshly broken. The space-range domain is indiscriminate to higher dimension for color-weighted bilateral filtering. The exclusive cost owing to the elevated dimensionality can be reduced by the Gaussian kd-trees, the Permutohedral Lattices, or the Adaptive Manifolds. But the performance of these methods is not competitive for grayscale bilateral filters because they use up a lot additional time preparing the data structures. Given the limitations of the bilateral filter, people began to explore new designs of fast edge-preserving filters.
The $\mathrm{O}(\mathrm{N})$ times Edge-Avoiding Wavelets (EAW) is wavelets with precise image-adaptive weights. But the kernel of the wavelets are sparsely distributed in the image plane, with constrain kernel range (to powers of two), which may limit the applications. Recently, Gastal and Oliveira recommend a auxiliary $\mathrm{O}(\mathrm{N})$ time filter acknowledged as the "Domain Transform filter". The key idea is to iteratively and separably apply $1 \mathrm{D}$ edge-aware filter. The $\mathrm{O}(\mathrm{N})$ time complexity is achieved by integral images or recursive filtering. We determine evaluate with this filter in this paper.

\section{B. Implicit Weighted-Average Filters}

A string of approach optimizes a quadratic cost function and solves a linear system, which is equivalent to implicitly filter a figure by an inverse matrix. In image segmentation and colorization, the affinities of this matrix are Gaussian functions of the shade similarity. In image matting, a matting Laplacian matrix is designed to enforce the alpha matte as a confined linear transform of the image colors. This matrix is also applied in haze removal. The weighted least squares filter in adjust the matrix resemblance according to the image gradients and produces halo-free edge-preserving smoothing. Although these optimization-based approaches often generate high quality results, solving the linear arrangement is prolonged. Direct solution like Gaussian Elimination is not practical due to the memory-demanding "filled in" predicament. Iterative solvers like the Jacobi method, Successive Over-Relaxation (SOR), and Conjugate Gradients are too slow to converge. Though carefully designed preconditioners greatly reduce the iteration number, the computational cost is still high. The multi grid method [is proven $\mathrm{O}(\mathrm{N})$ time complex for homogeneous Poisson equations, but its quality degrades when the matrix become extra inhomogeneous. Empirically, the implicit weightedaverage filters take at least a few seconds to progression a single megapixel picture either by pre-conditioning or by multigrid. It has been observed that these inherent filters are very much related to the explicit ones. In Elad shows that the bilateral filter is one Jacobi iteration in solution of the Gaussian affinity matrix. The Hierarchical Local Adaptive Preconditioners and the Edge-Avoiding Wavelets are constructed in a analogous mode. In this paper, we explain that the guided filter is closely related to the matting Laplacian matrix.

\section{Non average Filters}

Edge-preserving filter can also be attained by non average filters. The median filter is a well-known edge-aware operator, and is an exceptional case of narrow histogram filters. Histogram filters have $\mathrm{O}(\mathrm{N})$ time implementations in a way as the bilateral grid. The Total-Variation (TV) filter optimizes an L1-regularized cost function, and are shown equivalent to iterative median filtering. The $\mathrm{L} 1$ rate function may also be optimized by means of half-quadratic split, alternating between a quadratic model and spongy contraction (thresholding). In recent times, Paris et al. proposed manipulating the coefficients of the Laplacian Pyramid in the region of every pixel for edge-aware filtering. Xu et al. Propose optimizing an L0-fixed cost function favoring piecewise steady solution. The non average filters are often computationally expensive

\section{CONCLUSION}

This paper has reviewed the mainly latest research trends and proposed a weighted guided image filter (WGIF). In this paper WGIF is introduced by incorporating an edge-aware weighting into the guided image filter (GIF). In this paper we 
have presented the survey of weighted guided image filtering. In this paper proposed that local filtering-based edge preserving smoothing techniques.

\section{REFERENCES}

[1] Zhengguo Li, Jinghong Zheng, Zijian Zhu, Wei Yao, Shiqian $\mathrm{Wu}$ "Weighted Guided Image Filtering" IEEE Transactions on Image Processing, Vol. 24, No. 1, January 2015 1057-7149 @ 2014 IEEE.

[2] B. Y. Zhang and J. P. Allebach, "Adaptive bilateral filter for sharpness enhancement and noise removal," IEEE Trans. Image Process., vol. 17, no. 5, pp. 664-678, May 2008

[3] A. K. Moorthy and A. C. Bovik, "A two-step framework for constructing blind image quality indices," IEEE Signal Process. Lett. vol. 17, no. 5, pp. 513-516, May 2010.

[4] J. Pang, O. C. Au, and Z. Guo, "Improved single image dehazing using guided filter," in Proc. APSIPA ASC, Xi'an, China, 2011, pp. 1-4.

[5] P. Charbonnier, L. Blanc-Feraud, G. Aubert, and M Barlaud, "Deterministic edge-preserving regularization in computed imaging," IEEE Trans. Image Process., vol. 6, no. 2, pp. 298-311, Feb. 1997.

[6] Pierre Charbonnier, Laure Blanc-F'eraud, Gilles Aubert, Michel Barlaud "Deterministic Edge-Preserving Regularization in Computed Imaging" IEEE TRANSACTIONS ON IMAGE PROCESSING, VOL. 6, NO. 2, FEBRUARY 1997

[7] T. Mertens, J. Kautz, and F. Van Reeth, "Exposure fusion: A simple and practical alternative to high dynamic range photography," Comput. Graph. Forum, vol. 28, no. 1, pp. 161-171, 2009.
[8] K. Moorthy and A. C. Bovik, "A two-step framework for constructing blind image quality indices," IEEE Signal Process. Lett., vol. 17, no. 5, pp. 513-516, May 2010.

[9] L. I. Rudin, S. Osher, and E. Fatemi, "Nonlinear tota variation based noise removal algorithms," Phys. D, Nonlinear Phenomena, vol. 60, nos. 1-4, pp. 259-268, Nov. 1992

[10] Z. G. Li, J. H. Zheng, and S. Rahardja, "Detail-enhanced exposure fusion," IEEE Trans. Image Process., vol. 21, no. 11, pp. 4672-4676, Nov. 2012.

[11] R. Fattal, M. Agrawala, and S. Rusinkiewicz, "Multiscale shape and detail enhancement from multilight image collections," ACM Trans. Graph, vol. 26, no. 3, pp. 51:1-51:10, Aug. 2007

[12] R. C. Gonzalez and R. E. Woods, Digital Image Processing. Upper Saddle River, NJ, USA: Prentice-Hall, 2002.

[13] Levin, D. Lischinski, and Y. Weiss, "A closed-form solution to natural image matting," IEEE Trans. Pattern Anal. Mach. Intell., vol. 30, no. 2, pp. 228-242, Feb. 2008.

[14] J. Preetham, P. Shirley, and B. Smits, "A practical analytic model for daylight," in Proc. SIGGRAPH, 1999, pp. $91-100$.

[15] S. G. Narasimhan and S. K. Nayar, "Chromatic framework for vision in bad weather," in Proc. IEEE Conf. Comput. Vis. Pattern Recognit. (CVPR), Jun 2000, pp. 598-605. 